\title{
Lettre des éditeurs
}

Nota de les editores

Nota de las Editoras

Editors' Notes

DOI: http://dx.doi.org/10.5565/rev/jt13.722

\section{Lettre des éditeurs}

Nous initions ce premier volume de l'année avec un article du Dr Anna Camps. Cet article se fonde sur le discours prononcé par cette dernière pour l'inauguration du Master Officiel en Recherche en Éducation en 2016/17. Camps traite de l'évolution de la recherche en langue première et seconde pendant les 25 dernières années, en s'attachant plus particulièrement aux domaines d'études de son groupe de recherche, le GREAL (UAB). Camps est professeur émérite en enseignement - apprentissage des langues à l'UAB, elle a été une figure de proue de son domaine de recherche pendant les dernières décennies.

En tant qu'éditeurs, nous sommes heureux d'offrir un soutien, aussi petit soit-il, à nos collègues universitaires assiégés en Syrie, en initiant la section d'articles de recherche par un texte d'Abidrabbo Alnassan, qui propose une critique empirique des contenus lexicaux des livres de texte pour l'enseignement de l'arabe langue seconde dans une institution d'enseignement supérieur en Syrie. Cet article est suivi par l'étude d'Ana Fernández Viciana et Alberto Fernández Costales sur les perceptions de professeurs d'anglais en formation sur leur efficacité et leur préparation linguistique, à partir de données recueillies dans une université publique espagnole. Finalement, Laura Portolés Falomir et Otilia Martí Arnándiz explorent les pratiques translangagières dans l'apprentissage précoce des langues, en examinant des données qualitatives recueillies dans une école maternelle de Valencia.

Les articles sur la recherche sont complétés par une interview du Dr James Simpson, de la School of Education de l'Université de Leeds, menée et rédigée par Stefan Vollmer, étudiant en doctorat dans la même institution. Simpson et Vollmer traitent du TLang project, dans lequel Simpson est co-chercheur, ESOL, et de la relation entre la pratique de l'enseignement et la recherche.

Le volume s'achève para une présentation par Jessica Bradley de la collection Linguistic Ethnography: Interdisciplinary Explorations, éditée par Snell, Julia, Shaw, Sara et Fiona Copland. L'auteur réfléchit sur les articles de ce volume à partir de sa propre recherche transdisciplinaire sur le théâtre de rue multilingue.

Nous espérons une fois de plus que vous trouverez ce volume de Bellaterra Journal of Teaching and Learning Language and Literature à la fois informatif et inspirant.

Dr Melinda Dooly \& Dr Emilee Moore

31 Mars 2017

\section{Crédits:}

Les illustrations des couvertures de chaque numéro sont élaborées par des étudiants d'EINA (École de Design et d'Art, Barcelone) réalisant le cours de Post-grado « Illustration créative », sous la direction de Sonia Pulido, professeur d'illustration pour médias imprimés. 
Les traductions des notes des éditeurs ont été réalisées par l'Unitat d'Assessorament Lingüístic i Traduccions, Servei de Llengües, Universitat Autònoma de Barcelona.

\section{Réviseurs du Volume 10.1}

Les éditeurs voudraient remercier les réviseurs suivants pour leur contribution à la préparation du Volume 10.1:

Cristina Aliagas, Universitat Pompeu Fabra; Victoria Antoniadou, University of Nicosia Medical School; Laia Canals, Universitat Oberta de Catalunya/Universitat Autònoma de Barcelona; Xavier Fontich, Universitat Autònoma de Barcelona; Lynne Franjié, Université Charles de Gaulle - Lille 3; Carolin Fuchs, City University of Hong Kong; Robert O'Dowd, Universidad de León; Randall Sadler, University of Illinois at Urbana Champaign; Jöel Thibeault, University of Ottawa 


\section{Nota de les editores}

Comencem el primer volum de l'any amb un article convidat de la Dra. Anna Camps. La seva contribució es basa en la conferència inaugural de Camps per al Màster Oficial en Recerca en Educació del curs 2016/17. Camps se centra en l'evolució de la investigació en primera i segona llengua en els últims 25 anys, fent una atenció especial en les àrees d'investigació del seu grup de recerca, GREAL (UAB). Camps, és professora emèrita en Didàctica de la Llengua de la UAB, i ha estat una figura destacada en el seu camp de recerca de les últimes dècades.

Com a editores, ens alegra poder oferir suport, encara que sigui una petita contribució, als nostres col·legues acadèmics assetjats a Síria i, per això, la secció d'articles d'investigació comença amb un text d'Abidrabbo Alnassan, que ofereix una crítica basada en dades empíriques del contingut lèxic de llibres de text per a l'ensenyament de l'àrab com a segona llengua en una institució d'educació superior a Síria. Tot seguit, trobem l'estudi d'Ana Fernández Viciana i Alberto Fernández Costales, que se centra en les percepcions dels professors d'anglès en formació sobre la seva eficàcia i preparació lingüística abans de començar a treballar, analitzant dades recollides en una universitat pública espanyola. Finalment, Laura Portolés Falomir i Otilia Martí Arnándiz exploren pràctiques de translingüisme en l'aprenentatge primerenc de la llengua, a partir de l'anàlisi de les dades qualitatives recollides en una escola bressol valenciana.

Complementem els articles d'investigació amb una entrevista al Dr. James Simpson, de l'Escola d'Educació de la Universitat de Leeds, duta a terme i compilada per Stefan Vollmer, un estudiant de doctorat de la mateixa institució. Simpson i Vollmer parlen sobre el projecte TLang, del qual Simpson és co-investigador, l'ensenyament d'anglès com a segona llengua, i la relació entre la pràctica de l'ensenyament i la investigació.

Acabem el volum amb una ressenya de Jessica Bradley de la col·lecció Linguistic Ethnography: Interdisciplinary Explorations, editada per Snell, Julia; Shaw Sara i Fiona Copland. L'autora reflexiona sobre les aportacions del volum des del punt de vista del seu propi treball transdisciplinari en el teatre de carrer multilingüe.

Un cop més, esperem que es aquest volum del Bellaterra Bellaterra Journal of Teaching and Learning Language and Literature resulti informatiu i inspirador.

Dra. Melinda Dooly i el Dra. Emilee Moore

31 de de març de 2017

\section{Crèdits:}

Les il·lustracions per a les portades de cada número estan dissenyades pels estudiants d'EINA (Escola de Disseny i Art, Barcelona) que estudien en el curs de postgrau Il·lustració Creativa, sota la direcció de Sonia Pulido, professora d'Il·lustració per a Premsa.

Les traduccions de les notes de les editors han estat realitzades per la Unitat d'Assessorament Lingüístic i Traduccions, Servei de Llengües, Universitat Autònoma de Barcelona. 


\section{Revisors del Volum 10.1}

Les editores volen agrair als següents revisors la seva contribució a la preparació del Volum 10.1:

Cristina Aliagas, Universitat Pompeu Fabra; Victoria Antoniadou, University of Nicosia Medical School; Laia Canals, Universitat Oberta de Catalunya/Universitat Autònoma de Barcelona; Xavier Fontich, Universitat Autònoma de Barcelona; Lynne Franjié, Université Charles de Gaulle - Lille 3; Carolin Fuchs, City University of Hong Kong; Robert O'Dowd, Universidad de León; Randall Sadler, University of Illinois at Urbana Champaign; Jöel Thibeault, University of Ottawa 


\section{Nota de las editoras}

Empezamos el primer volumen del año con un artículo invitado de la Dra. Anna Camps. Su contribución se basa en la conferencia inaugural de Camps para el Máster Oficial en Investigación en Educación para el curso 2016/17. Camps analiza la evolución de la investigación en primera y segunda lengua en los últimos 25 años, centrándose especialmente en las áreas a las que se dedica su grupo de investigación, GREAL (UAB). Camps es profesora emérita de Didáctica de la Lengua en la UAB y ha sido una figura destacada en su campo de investigación durante las últimas décadas.

Como editoras, nos alegramos de poder ofrecer apoyo, aunque sea un pequeño gesto, a nuestros compañeros académicos en Siria, iniciando la sección de artículos de investigación con un texto de Abidrabbo Alnassan, que ofrece una crítica empírica del contenido léxico de los libros de texto para la enseñanza del árabe como segunda lengua en una institución de educación superior en Siria. A continuación, presentamos el estudio de Ana Fernández Viciana y Alberto Fernández Costales acerca de la percepción de los profesores de inglés antes de su servicio sobre su eficacia y preparación lingüística, a partir de datos recogidos en una universidad pública española. Por último, Laura Portolés Falomir y Otilia Martí Arnándiz exploran las prácticas de translingüismo en el aprendizaje temprano de lenguas, examinando datos cualitativos recogidos en un jardín de infancia valenciano.

Complementamos los artículos de investigación con una entrevista al Dr. James Simpson, de la Escuela de Educación de la Universidad de Leeds, realizada y compilada por Stefan Vollmer, un estudiante de doctorado de la misma institución. Simpson y Vollmer hablan sobre el proyecto TLang, del que Simpson es co-investigador, sobre el inglés como lengua extranjera, y sobre la relación entre la práctica docente y la investigación.

Terminamos el volumen con una reseña de Jessica Bradley de la Linguistic Ethnography: Interdisciplinary Explorations, editado por Snell, Julia; Shaw, Sara, y Fiona Copland. La autora reflexiona sobre las aportaciones del volumen desde el punto de vista de su propio trabajo transdisciplinar sobre el teatro callejero multilingüe.

Una vez más, esperamos que este volumen del Bellaterra Journal of Teaching and Learning Language and Literature resulte informativo e inspirador.

Dra. Melinda Dooly y Dra. Emilee Moore

31 de marzo de 2017

\section{Créditos:}

Las ilustraciones de las portadas de cada número han sido diseñadas por estudiantes de EINA (Escola de Disseny i Art, Barcelona), que cursan el posgrado Il·lustració Creativa, bajo la dirección de Sonia Pulido, profesora de Ilustración para Prensa.

Las traducciones de las notas de las editoras han sido realizadas por la Unitat d'Assessorament Lingüístic i Traduccions, Servei de Llengües, Universitat Autònoma de Barcelona. 


\section{Revisores para el Volumen 10.1}

Las editoras desean agradecer a los siguientes revisores su contribución a la preparación del Volumen 10.1:

Cristina Aliagas, Universitat Pompeu Fabra; Victoria Antoniadou, University of Nicosia Medical School; Laia Canals, Universitat Oberta de Catalunya/Universitat Autònoma de Barcelona; Xavier Fontich, Universitat Autònoma de Barcelona; Lynne Franjié, Université Charles de Gaulle - Lille 3; Carolin Fuchs, City University of Hong Kong; Robert O'Dowd, Universidad de León; Randall Sadler, University of Illinois at Urbana Champaign; Jöel Thibeault, University of Ottawa 


\section{Editors' Note}

We begin the first volume of year with an invited article from Dr. Anna Camps. The contribution is based on Camps' 2016/17 inauguration address for the Official Master's Degree in Research in Education. Camps discusses how research in first and second language has evolved over the past 25 years, focusing especially on those areas of enquiry addressed by her research group, GREAL (UAB). Camps is Emeritus Professor in Language Teaching and Learning at the UAB, and has been a leading figure in her field of research for the past several decades.

As editors, we are content to be able to offer support, albeit in a small way, to our beseiged academic colleagues in Syria by beginning the section on research articles with a text by Abidrabbo Alnassan, who offers an empirically founded critique of the lexical content of textbooks for teaching Arabic as a second language at a higher education institution in Syria. This is followed by Ana Fernández Viciana and Alberto Fernández Costales' study on preservice English teachers' perceptions of their efficacy and linguistic preparation using data collected at a public Spanish university. Finally, Laura Portolés and Otilia Martí explore translanguaging practices in early language learning, examining qualitative data collected in a Valencian kindergarten.

We complement the research articles with an interview with Dr. James Simpson, from the School of Education at the University of Leeds, conducted and compiled by Stefan Vollmer, a doctoral student from the same institution. Simpson and Vollmer discuss the TLang project, which Simpson is co-investigator on, ESOL, and the relationship between teaching practice and research.

We end the volume with a review by Jessica Bradley of the collection Linguistic Ethnography: Interdisciplinary Explorations, edited by Snell, Julia, Shaw, Sara and Fiona Copland. The author reflects on the contributions of the volume from the standpoint of her own transdisciplinary work on multilingual street theatre.

Once again, we hope you will find this volume of Bellaterra Journal of Teaching and Learning Language and Literature informative and inspiring.

Dr. Melinda Dooly \& Dr. Emilee Moore

31 March 2017

\section{Credits:}

Illustrations for the covers of each issue are designed by students of EINA (Escola de Disseny i Art, Barcelona) studying in the postgraduate course 'Il·lustració Creativa', under the direction of Sonia Pulido, teacher of Illustration for Publishing Media.

Translations of the editors' notes has been done by the Unitat d'Assessorament Lingüístic $\mathrm{i}$ Traduccions, Servei de Llengües, Universitat Autònoma de Barcelona. 


\section{Reviewers for Volume 10.1}

The editors would like to thank the following reviewers for their contribution to the preparation of Volume 10.1:

Cristina Aliagas, Universitat Pompeu Fabra; Victoria Antoniadou, University of Nicosia Medical School; Laia Canals, Universitat Oberta de Catalunya/Universitat Autònoma de Barcelona; Xavier Fontich, Universitat Autònoma de Barcelona; Lynne Franjié, Université Charles de Gaulle - Lille 3; Carolin Fuchs, City University of Hong Kong; Robert O'Dowd, Universidad de León; Randall Sadler, University of Illinois at Urbana Champaign; Jöel Thibeault, University of Ottawa

Pour citer cet article:

Dooly, M., \& Moore, E. (2017). Lettre des éditeurs, 10.1. Bellaterra Journal of Teaching \& Learning

Language \& Literature, 10(1), 1-8. DOI: http://dx.doi.org/10.5565/rev/jt13.722

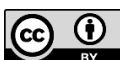

